\title{
EL PALEOLÍTICO SUPERIOR EN LA BAHÍA DE MÁLAGA: REFLEXIONES PARA UN NECESARIO DEBATE
}

\author{
THE UPPER PALAEOLITHIC IN THE BAY OF MÁLAGA. \\ REFLECTIONS FOR A NECESSARY DEBATE
}

por

\author{
JOSÉ RAMOS MUÑOZ *
}

\section{INTRODUCCIÓN}

Mi dedicación al estudio del Paleolítico Superior en el marco geográfico andaluz, viene motivada por mi vinculación personal con el Dr. Enrique Vallespí (Profesor de Prehistoria de la Universidad de Sevilla), que desde que tuve la suerte de trabajar con él, me animó a investigar en dicho momento histórico. Las circunstancias de la investigación me inclinaron a abordar estudios de Prehistoria Reciente, sobre todo en la perspectiva de las industrias líticas. Ello me posibilitó poder así tener una familiaridad con temas y técnicas de los conjuntos líticos del Neolítico y Edad del Cobre, tema sustancial de mi Tesis Doctoral (Ramos, 1986 y 1988).

Las excavaciones en el marco del Proyecto Porcuna (Jaén) (dirigidas por el profesor Dr. Oswaldo Arteaga, Director del Departamento de Prehistoria y Arqueología de la Universidad de Sevilla) nos posibilitaron en la campaña de 1991 la excavación de la Peña de la Grieta (Arteaga y otros, 1991) con la documentación de una completa estratigrafía de Paleolítico Superior Avanzado.

Además los estudios sobre la Cueva de Doña Trinidad (Ardales, Málaga), en colaboración con los compañeros Mar Espejo, Pedro Cantalejo y Emilio Martín (Ramos y otros, 1992; en prensa c) me pusieron en contacto directo con el arte paleolítico.

En los últimos años el proyecto de investigación del que tengo responsabilidad en la Universidad de Cádiz, titulado "Las ocupaciones prehistóricas de la campiña litoral y banda atlántica de Cádiz", debidamente autorizado y subvencionado por la Junta de Andalucía, nos ha permitido tras tres campañas de prospecciones (Ramos y otros, 1993, 1994, en prensa a, en prensa b) documentar asentamientos del Paleolítico Superior en el marco de formaciones de suelos rojos adscritos a la aridificación Soltaniense, en el marco de formaciones complejas de carácter edafo-sedimentario, con correlación genética y cronológica con la formación de "sables rouges" del N. de Marruecos, datadas allí en sus inicios entre 28.000-24.000 B.P. (Texier y Raynal, 1989; Debenath y otros, 1986; Nehren, 1992).

* Universidad de Cádiz. 
Estos hallazgos interesantes de la orla atlántica se completan con recientes descubrimientos en la Bahía de Algeciras de enclaves de Paleolítico Superior Final, en colaboración con Maria Isabel Arroquia y otros arqueólogos miembros del Instituto de Estudios del Campo de Gibraltar.

Esta continuidad de investigaciones en diversos puntos de la geografía andaluza, me permiten reflexionar sobre un área geográfica, a mi parecer fundamental para la comprensión global del Paleolítico Superior del Sur peninsular !.

\section{SUCINTO APUNTE HISTORIOGRÁFICO}

Importantes cuevas malagueñas, tuvieron la fortuna de entrar pronto, a principios de siglo en la historiografía europea, debido al interés y esfuerzo de los grandes investigadores sistematizadores del Paleolítico Occidental, como fueron Henri Breuil y Hugo Obermaier. Así los descubrimientos de las cuevas decoradas con arte, caso de Pileta (Benaoján) (Breuil y otros, 1915) o de Doña Trinidad (Ardales) (Breuil, 1921) indicaban que en el lejano sur, había un foco de ocupación paleolítico y artístico, comparable al área clásica de la Península Ibérica o a las grandes cuevas de la Dordoña o Perigord.

En aquella época la inquietud del insigne investigador, nunca suficientemente reconocido por la moderna historiografía, como fue Miguel Such, excavaba en 1917 y 1918, y publicaba con metodología moderna y europea, la Cueva del Higuerón (Such, 1920), que aportaba una secuencia de gran personalidad, con tres niveles: mixto, tardenoisiense y capsiense. Dicha secuencia y el magnífico estudio de Such, perduró más de 50 años y sirvió a Javier Fortea, para que en su brillante tesis doctoral, en la Universidad de Salamanca, plantease la posibilidad de un foco Magdaleniense en la Bahía de Málaga, donde sobre unas bases avanzadas de filiación Tardigravetiense, se fijaría una continuidad estratigráfica, hacia modos de vida locales epipaleolíticos y que tendrían manifiestas continuidades estratigráficas en la ocupación neolítica (Fortea, 1973 y 1986).

¿Qué pasó con la investigación del Paleolítico Superior en Málaga? ¿Por qué se interrumpieron los estudios?

Sin duda son preguntas que un detenido análisis historiográfico debe plantearse. Como en tantos otros temas de nuestra Arqueología Prehistórica, la Guerra Civil supuso un corte radical y las condiciones sociológicas de los años de la Post-guerra no permitieron gran cosa.

De este modo, los años 40 y 50 fueron de un largo secano en la investigación del Paleolítico Superior. En los 60 sólo destaca la poco novedosa aportación de Gimenez Reyna, que prácticamente transcribía al castellano el artículo de Breuil de 1921 (Gimenez, 1963 y 1964).

El descubrimiento en 1959 y las sucesivas investigaciones desarrolladas en Cueva de Nerja pusieron en evidencia ya desde los años 60 (Gimenez, 1962, G.e.s. de.la S.e.m., 1985; Durán, 1994), la importante documentación de un complejo santuario de arte paleolítico (Sanchidrián, 1986) y de un asentamiento de rica

1. Quiero destacar mi agradecimiento hacia el profesor Enrique Vallespí, que tantas horas dedicó a mi formación, y que fomentó siempre en mí la necesidad de «reflexionar sobre el Paleolítico».

Quiero también agradecer la constante ayuda del profesor Oswaldo Arteaga, con quien tuve la suerte de formarme en arqueología de campo, por haberme instado siempre a «comprender la Historia como un proceso».

Extiendo mis agradecimientos a mis amigos y compañeros de Málaga, Pedro Cantalejo, Mar Espejo, Emilio Martín y Angel Recio, por su apoyo y constante colaboración.

Por fín destacar la ayuda continuada de los arqueólogos de Cádiz, Vicente Castañeda, Manuela Pérez, María Lazarich, Cristina Martínez, Manuel Montañés, Maria Isabel Arroquia, Luis Pérez, Maria Isabel Gómez, por su colaboración en la documentación y estudio del Paleolítico Superior de la Banda Atlántica de Cádiz, así como a Francisco Martínez. 
secuencia estratigráfica (Jordá, 1986), que en la interpretación del maestro Francisco Jordá, en una magnífica monografía interdisciplinar, expone desde un inicio, la secuencia en el Auriñaciense, con Solutrense, Magdaleniense de gran personalidad y modesto Epipaleolítico, que conectaría con una magnífica continuidad ocupacional en la Prehistoria Reciente (Jordá, 1985 y 1986).

La importancia de Cueva de Nerja radica en la confirmación de una amplia secuencia de ocupación, con un modelo continuado de frecuentación que ha permitido la posibilidad de estructuración cronoestratigráfica de las estaciones del Paleolítico Superior de la Bahía de Málaga.

El magisterio desempañado por el Profesor Jordá dejó huellas fundamentales para la Prehistoria de Málaga. En los años 70, la Tesis de Javier Fortea, titulada en su publicación Los complejos microlaminares y geométricos del Epipaleolítico mediterráneo español (Fortea, 1973), venía a plantear la existencia de un foco Magdaleniense en la Bahía de Málaga, de conexión mediterránea, enmarcado en la sucesión de las facies microlaminar, postmagdaleniense y geométrica con clara continuidad neolítica. Sí quedaba claro el reto planteado entonces por Fortea "Tras ese mal conocido Magdaleniense, cuya presencia en Málaga señala una importante tarea por aclarar con posibles repercusiones en el arte parietal de esta provincia..." (Fortea, 1973: 500). En dicho contexto Magdaleniense englobaba Fortea, los materiales de Cuevas como Hoyo de la Mina, Cueva del Higuerón, o Cueva de la Victoria, con sustantiva industria ósea de 2 arpones de 1 hilera en Cueva de la Victoria, 1 arpón en la Cueva del Higuerón y la destacada serie de buriles, laminitas con borde abatido, 1 triángulo, láminas y laminitas con muesca y denticulados, de Hoyo de la Mina, en el denominado Nivel Capsiense de Such (Fortea, 1973).

Los años 70 suponen también la integración de las grandes cavidades con arte de Málaga en síntesis amplias de carácter macro-regional (Jordá, 1978; Fortea, 1978; Graziosi, 1974). Igualmente se descubren nuevas cavidades con arte, caso de Cueva del Toro o Calamorro (Fortea y Gimenez, 1972-1973).

Los 80 suponen la entrada en escena de jóvenes investigadores formados en la Universidad de Málaga, que realizaron investigaciones con el profesor Jordá en Nerja, caso de José Luis Sanchidrián, Cecilio Barroso y Julián Ramos. De hecho estos últimos años representan un desarrollo sustantivo, con nuevas excavaciones y descubrimientos destacados.

En principio, las excavaciones de Cecilio Barroso en Cueva del Boquete de Zafarraya (Alcaucín) vinieron a conformar junto con Jean Jacques Hublin, un equipo interdisciplinar que está sentando bases importantes sobre la continuidad de los Neandertales y la importante personalidad de la transición que en el Sur peninsular manifiestan los Sapiens Neandertalensis y probablemente la coetaneidad de ocupaciones entre Neandertales y Sapiens (Barroso y otros, 1983 y 1993; Barroso y Hublin, 1994). Sus investigaciones ofrecen luz al modo de ocupación en el inicio del Paleolítico Superior y a sugestivos problemas de transición antropológica y tecnológica.

Igualmente creo importantes las excavaciones de Julián Ramos en Cuevas de la Cala del Moral (aún sin publicar).

La nueva documentación de hallazgos de arte paleolítico corrió a cargo de dos grupos de trabajo. Por una parte, de José Luís Sanchidrián que documentaba en 1981 la magnífica Cueva Navarro (Málaga) (Sanchidrián, 1981), realizaba estudios en Ardales (Sanchidrián, 1994), Nerja (Sanchidrián, 1986a) y Pileta (Sanchidrián, 1986b) que cristalizan en síntesis de interpretación estructuralista, basadas en estructuras topo-iconográficas, asociaciones de las figuras y ubicaciones microespaciales (Sanchidrián, 1991-1992 y 1994).

Por otro lado, el equipo constituido por Mar Espejo y Pedro Cantalejo, que realizan aportaciones en Cueva del Higuerón (Espejo y Cantalejo, 1987 y 1989) y en Cueva de Ardales (Espejo y Cantalejo, 1988; Ramos y otros, 1992). De estos estudios, se confirmaba la asociación de hábitat y santuario en el complejo de Higuerón y se replanteaba la importancia de Cueva de Ardales y las relaciones que estos santuarios del interior tienen con el foco de ocupaciones costeras (Ramos y otros, en prensa c). 
Paralelamente y de modo sorprendentemente más modesto que la aparición de los enclaves con arte, se han ido documentando nuevos lugares de hábitat, producto más del azar que de una investigación continuada, lo que nos confirma la acertada apreciación de Fortea de "lo anormal que resultaba el magro conocimiento que tenemos de las industrias paleolíticas frente a algo tan serio como el arte paleolítico andaluz. Su sola presencia está indicándonos que los depósitos arqueológicos conocidos son sólo una parte mínima de la nutrida y variada red que encontraremos, una vez que se potencien las tareas de prospección" (Fortea, 1986: 76).

Nos referimos a la documentación de material Solutrense en Cueva del Higuerón (Rincón de la Victoria) (Lopez y Cacho, 1979), Cueva del Tajo de Jorox (Alozaina) (Marques y Ruiz, 1976) y la más reciente de El Bajondillo (Torremolinos) (Marques y Ferrer, 1991).

Otras prospecciones desarrolladas en la cuenca del río Vélez, en su curso alto (ríos Alcaucín, Guaro, Sábar, de la Cueva) a cargo de José Ramos, Emilio Martín y Angel Recio documentaron una importante continuidad de los sustratos superopaleolíticos, en los conjuntos líticos del Neolítico y permitieron localizar abrigos con material mezclado en superficie de significativa relevancia, por un lado de ocupaciones neolíticas y calcolíticas, pero también con posibilidad de potencia estratigráfica, caso de Cueva del Espino (Alcaucín), aquí en colaboración con Alvaro Moreno; Abrigos del Tajo Doña Ana, Abrigo del Cortijo de la Cueva, Cueva de la Morronguera (Ramos, 1986, 1988 y 1988-1989; Martín, 1988, 1989 y 1995).

En síntesis debemos destacar de este panorama historiográfico, los brillantes orígenes con la intervención directa en los primeros estudios, de investigadores de la talla de Henri Breuil o Hugo Obermaier, y la dedicación local de Miguel Such que sentaron bases fundamentales en una secuencia que quedó en el olvido con la Guerra Civil y la Postguerra. Los hallazgos fueron puntuales en los años 60 y 70, donde destacó la gran aportación de Cueva de Nerja, a cargo de Francisco Jordá y la brillante tesis de Javier Fortea; señalando que en los últimos años, sólo aparecen hallazgos sueltos, de modo intermitente, e interesantes aportaciones al arte rupestre.

Lamentablemente no se continuó el modelo de investigación planteado por Fortea, y sólo Nerja, en constante y titánica labor de Jordá y equipo de la Universidad de Salamanca, han venido demostrando en los últimos años, la continuada secuencia, con un hábitat de ocupación dilatada, la personalidad del Magdaleniense y la perduración tecnológica del Epipaleolítico. Además confirma que en las cuevas con arte, los llamados santuarios, se ubican en zonas internas, existiendo salas de hábitat, con potentes estratigrafías, y que al menos teóricamente puede haber relación entre determinadas fases del hábitat y los autores de las pinturas.

Por tanto han sido pocos los equipos científicos consolidados que han actuado, destacando, el de la Universidad de Salamanca con la intervención directa de Francisco Jordá y sus discípulos, centrados en la investigación modélica ofrecida en Cueva de Nerja.

\section{LAS IMPORTANTES BASES HISTÓRICAS DE LA SECUENCIA}

Una buena parte de prehistoriadores españoles al menos hasta la década de los 80 , ha tenido una fuerte dependencia en la estructuración del Paleolítico Superior conforme al modelo de la secuencia clásica francesa. Sin duda la importante aportación historiográfica desde mediados del siglo XIX dejó modelado un esquema de secuencia desde Jouannet y su "Calendrier de la Dordogne" y la fijación de integración de estudios paleontológicos y geológicos en la obra de Boucher de Perthes : "Antiquités Celtiques et Antediluviennes" publicada entre 1847 y 1860 . Los aportes sustanciales desde el campo paleontológico de E. Lartet-H. Christy; de Gabriel de Mortillet con su "Formaticon de la Nation Française" y E. Piette con "L'art pendant l'age du renne" fueron decisivos (Laming-Emperaire, 1968). Pero fue sobre todo la figura genial y lúcida del Abate Henri Breuil quien sistematizó la secuencia clásica en el famoso artículo "Les subdivisiones du Paléolithique 
Supérieur et leur signification". Los aportes de Peyrony y de la escuela Bordes, separaron el bloque Auriñaciense del Perigordiense, pero al cabo dejaron fijado el modelo paradigmático (Breuil y Lantier, 1959; Bordes, 1968, 1984; Sonneville-Bordes, 1960).

Aparte de la suerte inicial en la investigación de que fueran autores de los primeros hallazgos, investigadores del prestigio de Breuil y Obermaier; para la fijación de la secuencia, se tuvo también la suerte, en la Bahía de Málaga, de la gran dedicación de Jordá a la Prehistoria de la Cueva de Nerja y al estudio de las cavidades decoradas de la provincia (Jordá, 1978, 1985, 1986). Ello ha incidido que la gran personalidad y manera de entender la secuencia de Jordá genere un modelo de personalidad suficiente y que no haya intentado ser "fijada con calzador" sobre modelos exógenos.

De todos modos, las investigaciones recientes que venimos de comentar, sugieren unas reflexiones sobre las bases documentales y al menos conceptualmente valorar en un marco amplio los datos de que disponemos.

El gran organigrama con que se cuenta procede lógicamente de Cueva de Nerja, que permitió al equipo del profesor Jordá fijar la primera ocupación de la Cueva en el Auriñaciense sensu lato, observando que "culturalmente estos tres niveles N.M.17, N.M.18 y N.M.19 son de difícil atribución" (Jordá, 1986:198). Sobre dichas ocupaciones de complejo encuadre se superpone un Solutrense Medio de facies ibérica, con hojas de laurel (de las excavaciones de la Sra. de la Quadra Salcedo) y por el conjunto industrial de los niveles N.V.10 y N.V.9. Sobre ellos los niveles N.M.16, N.M.15, y N.M.14, manifiestan una de las grandes etapas de la Cueva, con un Magdaleniense de gran personalidad, muy rico en hojitas, con mayor presencia de buriles que de raspadores, con buenas series de hojitas con muescas y denticulados, así como con destacada importancia de laminitas de borde abatido, constatándose el inicio del microlitismo geométrico. Todo ello acompañado de una rica industria ósea. En el marco del Magdaleniense Superior Mediterráneo Jordá siempre señaló la personalidad de estos conjuntos y su vinculación con los modos de vida de Cueva de Nerja. Sobre estos niveles viene una ocupación Epipaleolítica documentada en N.M.13 y N.M.12, que se enmarcan en una línea evolutiva del Magdaleniense y en un sentido amplio se adscriben a la Facies Microlaminar del Epipaleolítico Mediterráneo (Jordá, 1986).

Por otro lado, la excavación de Cueva del Boquete de Zafarraya ha deparado una perduración inesperada de los Neandertales más allá del 35.000 B.P. (Barroso y Hublin, 1994) y plantea diversas hipótesis, por un lado un cierto refugio de los Neandertales en el Sur peninsular y por otro una posible convivencia con los Sapiens Sapiens.

El resto de los hallazgos industriales sueltos vienen a llenar determinados lugares del organigrama de base, planteado por Cueva de Nerja. Cabe confirmar la atribución Postpaleolítica (Ramos, 1986) del conjunto de El Chorro (El Burgo), que había sido atribuido por sus autores al Auriñaciense (Ruiz y Leiva, 1980).

Todo lo expuesto nos deja un inicio claro de la secuencia en el Solutrense, quedando como importante problema la base de la ocupación de Nerja, de necesaria definición en los momentos iniciales del Paleolítico Superior. La escasez del conjunto y su propia problemática ya plantearon a Jordá, dificultades en su adscripción que, con su lucidez manifiesta observó claramente en relación a los enclaves auriñacienses del área mediterránea" que todos ellos ofrecen características culturales y morfológicas muy distintas entre sí que dificultan su adscripción a fases determinadas y específicas del Auriñaciense francés y aún cantábrico" (Jordá, 1986: 199).

Así en criterios normativos se adscriben al Solutrense los niveles N.V.10 y N.V.9 de Cueva de Nerja (Jordá, 1986), Cuevas del Higuerón (López y Cacho, 1979), Cueva del Tajo del Jorox (Marques y Ruiz, 1979), y El Bajondillo, que se constituye como un importante enclave costero sobre el travertino situado en Torremolinos, que sugiere una personalidad manifiesta en el Solutreogravetiense de raiz ibérica, con utillaje de dorso abatido y puntas de muesca (Marques y Ferrer, 1991).

Al Magdaleniense de amplia adscripción mediterránea quedan los niveles de Nerja N.M.16, N.M.15, N.M.14 
(Aura, 1986), Hoyo de la Mina, en el nivel Capsiense de Such (Such, 1920; Fortea, 1973); así como los arpones e indicios de las Cuevas de Higuerón (Gimenez y Laza, 1962) y Victoria (Ripoll, 1970), así como una azagaya de Cueva Tapada (Fortea, 1986).

Sobre estas bases, a todas luces modestas, dada la importancia de los yacimientos, y de la topografía de Málaga, con grandes vías de comunicación costa-interior, destaca la presencia de enclaves con arte rupestre paleolítico en la Bahía de Málaga: en Cueva de Nerja (Sanchidrián, 1986), Cueva Navarro (Sanchidrián, 1981), Complejo de Cuevas del Higuerón (Espejo y Cantalejo, 1988b), Cueva del Toro (Fortea y Gimenez, 1972-1973), Cueva de Pecho Redondo (Espejo y Cantalejo, 1988b). En el interior en el curso del Guadalhorce alto, Cueva de Ardales (Breuil, 1921; Gimenez, 1964; Espejo y Cantalejo, 1988a; Sanchidrián, 1994; Ramos y otros, en prensa c), y en la vía del Guadiaro, en conexión con el foco de Gibraltar y la Bahía de Algeciras, Cueva de Pileta (Sanchidrián, 1986) y Cueva del Gato.

\section{REFLEXIONES Y PROBLEMAS}

Al abordar los datos y secuencias del Paleolítico Superior de Málaga en un contexto regional valoramos con mayor amplitud la sustancial problemática que han aportado los investigadores que historiográficamente nos han precedido.

Para nosotros, el Sur de la Península Ibérica funcionó como un auténtico lugar de refugio durante la primera mitad del Paleolítico Superior. Ello condicionó una secuencia cultural y unos modos de vida, en el marco de la caza-recolección, diferentes a otras regiones de la Península Ibérica, por ejemplo la Zona Cantábrica, donde condiciones de clima más duras permitieron la presencia de especies faunísticas de clima frío.

En Andalucía, el estudio de la tecnología, en el marco de un "continuum" (Laplace, 1966, 1973) tecnológico, evidencia unos inicios de necesaria definición, que no pueden estar sujetos a los modelos del Cantábrico, ni franceses. Es decir que nos encontramos aún en un momento de la investigación que debe caracterizarse por la fijación de la secuencia y estructuración cronoestratigráfica. Por ello creo que problemas vinculados, con movilidad, estacionalidad, organización de las estrategias de caza, estructuración y agregación de las comunidades en los santuarios son aún dificiles de plantear, dadas las limitaciones del registro.

Ello tiene una base importante en la falta de tradición de estudios del Paleolítico Superior, a excepción de la labor de campo continuada por Jordá y las reflexiones sustantivas del propio Jordá (Jordá, 1978, 1985 y 1986), de Fortea (Fortea, 1973 y 1986) y Vallespí (Vallespí, 1986 y en prensa).

No se han consolidado equipos de excavadores locales, y los arqueólogos interesados en estos problemas debieron acudir a colaboraciones con organismos extraprovinciales, como Barroso con el Museo del Hombre de París; Sanchidrián con la Universidad de Salamanca o Ramos, Espejo, Cantalejo, Martín, con el Departamento de Prehistoria y Arqueología de la Universidad de Sevilla.

Además la falta de prospecciones sistemáticas en enclaves kársticos ha desembocado a esta situación modesta de conocimientos, a pesar de las posibilidades de futuro que ofrece el Paleolítico Superior de la Bahía de Málaga.

Por otro lado, la urbanización galopante desarrollada en la costa en los años 60 , sobre todo en el travertino de Torremolinos (Durán y otros, 1988), y en depósitos de arenas rojas adscritos en sentido amplio al Pleistoceno Final, entre Marbella y la Bahía de Algeciras, han debido suponer una verdadera destrucción de enclaves.

Otro tema de necesario debate "social" es la repercusión de la gestión, conservación-visita y su posible incidencia en las cavidades con arte rupestre.

Con las bases historiográficas de partida, con la rica e importante secuencia de Cueva de Nerja, con los 
grandes santuarios de arte paleolítico, y con la serie dispersa de pequeños enclaves que cuadran bien con la secuencia normativa, quiero esbozar simplemente una serie de grandes problemas que concernientes al Paleolítico Superior del Sur peninsular, podrían tener un campo de experimentación y contrastación con los estudios en los yacimientos del ámbito de Málaga.

1. La Transición de Neandertales a Sapiens Sapiens, plantea una personalidad específica a la secuencia local, desde el marco de zona de refugio, para grupos que aún vivían con esquemas tecnológicos previos a la leptolitización y a las técnicas de los cazadores especializados. Ello sugiere en momentos determinados una cohabitación de los espacios por comunidades de Neandertales y de Sapiens Sapiens, en los momentos finales de vida de aquellos, y plantea al menos conceptualmente interesantes problemas sobre convivencia, aculturación, enculturación, relaciones de dependencia. Pienso que modelos de explicación como los planteados por Gerhard Bosinski o Marcel Otte en sentido amplio para Centroeuropa, de difusión para el Auriñaciense, de aculturación para el Chatelperroniense o de invención de industrias con puntas foliáceas de la llanura del norte de Europa (Bosinski, 1967; Otte, 1985 y 1990), generan un debate que debemos aplicar al sur. Sólo que aquí entiendo la aculturación y la invención propias como modelos de explicación de las primeras industrias del Paleolítico Superior, sobre bases propias tecnológicas del mundo musteriense.

2. La personalidad de la secuencia, que en el marco de un encuadre mediterráneo, permite poner en duda el esquema clásico francés de Chatelperroniense, Auriñaciense, Perigordiense, cuestionando un vacío de 15.000 años, hasta el Solutrense que debe quedar cubierto por grupos locales de gran personalidad, como ya señalara Jordá, de sus niveles inferiores de Nerja, que requieren una fijación cronoestratigráfica, analizados desde el sur y en relación a los problemas planteados en la propuesta 1.

3. La fijación de la secuencia Solutrense. Jordá siempre destacó la importancia de las hojas de laurel de las excavaciones de la Sra. De la Cuadra en la Sala del Vestíbulo de Cueva de Nerja (Jordá, 1986). Dicha ocupación se confirmó en N.V.9 y N.V.10, pero no con la riqueza y problemática que todos esperamos de Nerja. El Bajondillo, Cueva del Higuerón, o la Cueva del Tajo del Jorox, parecen apuntar que momentos evolucionados de la secuencia del Solutreogravetiense, alcanzan un papel destacado. Habrá que fijar la posición funcional de dichos enclaves y definir su cronoestratigrafía. Pienso que ello será imposible en Tajo del Jorox y en Cueva del Higuerón, dado el impresionante saqueo y destrucción sufrido por ambas cuevas, de ahí la importancia actual de El Bajondillo.

Problemas como el origen del Solutrense, la vinculación al mundo Ateriense o la continuidad de modelos locales desde el Paleolítico Medio residual de larga tradición deben ser abordados. Además aquí se deberá incidir en los modelos sociales de agregación (Testard, 1966, 1985; Duhard, 1993; Bosinski, 1979 y 1992; Conkey, 1991) de los santuarios, en los fenómenos de posible frecuentación cíclica de algunos hábitats (Arteaga y otros, 1991), y en la delimitación de espacios de control de ciertas comunidades.

La vinculación con los importantes enclaves estratificados en Cueva Ambrosio Nivel II (Ripoll, 1986 y 1988), el estrato II de Les Mallaetes (Fortea y Jordá, 1976) el tramo 4'5-4'75 de Parpalló (Pericot, 1942), junto a los nuevos hallazgos posiblemente sincrónicos del ámbito andaluz, caso de Peña de la Grieta (Porcuna, Jaén) (Arteaga y otros, 1991), El Pirulejo (Priego, Córdoba) (Asquerino, 1988 y 1992) o los hallazgos del río Guadalete (Giles y otros, 1993), Gibraltar (Giles y otros, 1994), o la Banda Atlántica de Cádiz (Ramos y otros, 1994) plantean una relación macroespacial amplia con los enclaves del Solutreogravetiense de la Bahía de Málaga y un encuadre histórico, que en sí contiene todo un amplio mundo de desarrollo, sobre todo pensando en las trayectorias de movilidad de las comunidades, si se fijan criterios básicos de materias primas y estacionalidad en relación a los tipos de fauna. 
Pienso que los modelos metodológicos seguidos por los prehistoriadores alemanes en el Rhin o Donau serían de posible aplicación (Bosinski, 1979 y 1992; Weniger, 1982, 1989, 1990 y 1991) ${ }^{2}$.

4. La fijación de las relaciones del arte con la secuencia tecnológica es fundamental (Espejo y Cantalejo, 1988b; Sanchidrián, 1994). La confirmación de la adscripción Solutrense de los santuarios de Nerja, Pileta o Ardales puede ayudar a la comprensión espacial de dichos enclaves y valorar las vías de los ríos, Vélez, Guadalhorce o Guadiaro, como verdaderos caminos en la frecuentación estacional de asentamientos, permitiendo el establecimiento de unas relaciones costa-interior hasta hace poco insospechadas.

5. En relación con los enclaves de arte, la necesaria contrastación de la fauna de los lugares de hábitat, con los motivos representados en el arte sugiere que a pesar del máximo glacial de los entornos del 18.000 B.P., la zona sur y mediterránea debió contar con un enmarque climático peculiar, y favorable para el asentamiento humano (Jordá, 1986: 333 y ss.), como lo demuestra el predominio de cápridos, équidos y bóvidos en las representaciones de arte rupestre en las cuevas andaluzas (Sanchidrián, 1986a, 1986b y 1994; Espejo y Cantalejo, 1988a y 1988b; Ramos y otros, en prensa c).

6. El problema de la sucesión de las bases tardigravetienses, con series importantes de industrias de dorsos abatidos (perfectamente constatadas en Nerja en el Magdaleniense Superior) (Aura, 1986), en sus orígenes probablemente Solutrenses de la secuencia local y devenir, hacia el Magdaleniense, de tan fuerte personalidad, sugieren explicaciones desde la tecnología y desde los modos de vida, del importante papel de los enmangamientos de hojitas de dorsos, con retoques abruptos, con muescas y denticulados espesos, y su incidencia en las técnicas de la caza.

Esta presencia que arrancará al menos desde el Solutrense va a dar personalidad hasta el Epipaleolítico (González-Tablas, 1986) y se verá residualmente en los sustratos de los conjuntos neolíticos (Ramos, 1988-1989; Martín, 1988, 1989 y 1995).

7. La continuidad de los modos tecnológicos del Magdaleniense Final hacia el Epipaleolítico, que dentro de la gran personalidad tardimagdaleniense, deben ofrecer multitud de variantes y modelos locales, que sin duda no cuadran con los esquemas amplios fijados en las Facies microlaminar y geométrica del Mediterráneo español.

Creo en síntesis que aún debemos terminar de fijar la secuencia y que se deberán delimitar las líneas de investigación, no sólo a los aspectos tecnológicos y cronoestratigráficos, sino también a la profundización en los modos de vida (Bate, 1986 y 1989) y en las formas económicas y sociales de los cazadores y recolectores que frecuentaron de un modo permanente, durante más de 20.000 años la Bahía de Málaga $\mathrm{y}$ las tierras del interior.

2. La posibilidad de disfrute de una beca del Instituto Arqueológico Alemán en el verano de 1993 en diversos centros de investigación de Alemania fue decisiva para mi formación y mi concepción del Paleolítico Superior. Quiero destacar las gestiones y apoyos del Dr. Hermanfried Schubart, entonces Director del Instituto Arqueológico Alemán de Madrid; al director de la Kommission für Allgemeine und Vergleichende Archäologie de Bonn, Dr. Wolfgang W.Wurster, el apoyo y facilidades prestadas en mi estancia, así como a los colegas de dicha institución, en especial al Dr. Joseph Eiwanger y al Dr. Heiko Prümmers. En el Forschungsbereich Altsteinzeit Schloss Monreposs de Neuwied pude disfrutar de la magnífica biblioteca y de la formidable acogida del profesor Dr. Gerhard Bosinski y sus colaboradores, Dr. Michael Baales, Dr. Martin Street, Dr. Jürgen Vollbrecht, Dr. Joachim Schäffer. Agradecer al Dr. Horst Schulz del Geowissenschaften der Universität Bremen la magnífica acogida en Bremen. Y destacar la amistad y especial atención del Dr. Gerd C. Weniger en mi visita al Institut für Urgeschichte, der Universität Tübingen, que me mostró los principales enclaves del Donau y Baden-Würtemberg. A todos ellos mi más entrañable y agradecido recuerdo. 


\section{BIBLIOGRAFÍA}

ARTEAGA, O., RAMOS, J., ROOS, A.M. y NOCETE, F. (1991): "Balance a medio plazo del "Proyecto Porcuna". Campaña de 1991. Anuario Arqueológico de Andalucía. 1991. Actividades Sistemáticas. Consejería de Cultura. Junta de Andalucía. Sevilla.

ASQUERINO, M.D. (1988): “Avance sobre el yacimiento magdaleniense de "El Pirulejo" (Priego de Córdoba)”. Estudios de Prehistoria Cordobesa 4: 59-68. Universidad de Córdoba.

- (1992): El Pirulejo. Excmo. Ayuntamiento de Priego de Córdoba.

AURA, J.E. (1986): "La ocupación magdaleniense de la Cueva de Nerja (La Sala de la Mina)". En JORDÁ, F. y PELLICER, M.: La Prehistoria de la Cueva de Nerja (Málaga). Trabajos sobre la Cueva de Nerja no 1: 205-267. Patronato de la Cueva de Nerja. Málaga.

BARROSO, C., GARCÍA, M., RUIZ, A., MEDINA, F. y SANCHIDRIÁN, J.L. (1983): “Avance al estudio cultural, antropológico y paleontológico de la Cueva del "Boquete de Zafarraya" (Alcaucín, Málaga)". Antropología y Paleoecología Humana 3: 3-9. Granada.

BARROSO, C., HUBLIN, J.J. y MEDINA, F. (1993): "Zafarraya y el remplazamiento de los Neandertales por el hombre moderno anatómicamente en Europa Occidental (objetivos del Proyecto General de Investigación y resumen de los resultados obtenidos en las campañas de 1990-1993)". Investigaciones Arqueológicas en Andalucía: 1985-1992. Proyectos: 229-238. Junta de Andalucía. Huelva.

BARROSO, C. y HUBLIN, J.J. (1994): "The Late Neanderthal site of Zafarraya (Andalucía, Spain)". Gibraltar during the Quaternary. AEQUA Monografias 2: 61-70. Sevilla.

BATE, L.F. (1986): "El modo de producción cazador recolector o la economía del salvajismo". Boletín de Antropología Americana 13: 5-31. México.

- (1989): "Notas sobre el materialismo histórico en el proceso de investigación arqueológica". Boletín de Antropología Americana 19: 5-30. México.

BORDES, F. (1968): El mundo del hombre Cuaternario. Ediciones Guadarrama. Madrid.

- (1984): Leçons sur le Paléolithique. Tome II Le Paléolithique en Europe. Cahiers du Quaternaire nº 7. C.N.R.S. Paris.

BOSINSKI, G. (1967): Die Mittelpaläolithischen funde im Westlichen Mitteleuropa Monographien zur Urgeschichte 4. Köln.

- (1979): Die Ausgrabungen in Gönnersdorf 1968-1976 und die Siedlungsbefunde der Grabung 1968. In Der Magdalénien-Fundplatz Gönnersdorf. Band 3. Franz Steiner Verlag. Wiesbaden.

- (1992): Eiszeitjäger im Neuwieder Becken. Archäologie des Eiszeitalters am Mittelrhein. Archäologie an Mittelrhein und Mosel 1. Koblenz.

BREUIL, H. (1912): "Les subdivisions du Paléolithique supérieur et leur signification”. Anthropo. Archéo. Préhist., 14ème session, T. I: 165. Gèneve.

- (1921): “Nouvelles cavernes ornées paleolithiques dans la province de Málaga". L'Anthropologie XXXI: 239-243. Paris.

BREUIL, H., OBERMAIER, H. y WERNER, W. (1915): La Pileta à Benaoján. I.P.H. Mónaco.

BREUIL, H. y LANTIER, R. (1959): Les hommes de la pierre ancienne. $2^{2}$ Edición. Payot. Paris.

CONKEY, M.W. (1991): "Contexts of Action, Contexts for Power: Material Culture and Gender in the Magdalenian". En GERO, J.M. y CONKEY, M.W. (eds.): Enjendering archaeology. women and Prehistory Oxford.

DEBENATH, A., RAYNAL, J.P., ROCHE, J., TEXIER, J.P. y FEREMBACH, D. (1986): "Stratigraphie, habitat, typologie et devenir de l'Aterien Marocain: Donnés Récentes”. L'Anthropologie 90: 233-246. Paris.

DUHARD, J.P. (1993): "Upper Palaeolithic figures as a reflection of human morphology and social organization”. Antiquity 67: 83-91. Londres. 
DURÁN, J.J. (1994): Cueva de Nerja. Patronato de la Cueva de Nerja. Málaga.

DURÁN, J.J., GRÜN, R. y SORIA, J.M. (1988): "Edad de las formaciones travertínicas del flanco meridional de la Sierra de Mijas (provincia de Málaga, Cordilleras Béticas)". Geogaceta 5:61 y ss. Madrid.

ESPEJO, M., ESPINAR, I. y CANTALEJO, P. (1986): "Documentación gráfica del arte rupestre existente en el complejo subterráneo del Higuerón. Rincón de la Victoria (Málaga)". Anuario Arqueológico de Andalucía 1986. II Actividades Sistemáticas: 495-499. Consejería de Cultura. Junta de Andalucía. Sevilla.

ESPEJO, M. y CANTALEJO, P. (1988a): "La Cueva de Ardales: yacimiento recuperado". Revista de Arqueologia 84: 14-24. Madrid.

- (1988b): "Nuevas aportaciones al corpus artístico paleolítico del extremo occidental del Mediterráneo". Primer Congreso Internacional "El Estrecho de Gibraltar": 131-146. U.N.E.D. Madrid.

FORTEA, J. (1973): Los complejos microlaminares y geométricos del Epipaleolítico mediterráneo español. Memorias del Seminario de Prehistoria y Arqueología n⿳0 3. Universidad de Salamanca.

— (1978): “Arte Paleolítico del Mediterráneo español”. Trabajos de Prehistoria 35: 99-149. Madrid.

- (1986): "El Paleolítico Superior y Epipaleolítico en Andalucía. Estado de la cuestión cincuenta años después". Actas del Congreso Homenaje a Luis Siret (1934-1984): 67-78. Cuevas del Almanzora. Junio 1984. Consejería de Cultura. Junta de Andalucía. Sevilla.

FORTEA, J. y GIMENEZ, M. (1972-1973): "La Cueva del Toro. Nueva estación malagueña con arte Paleolítico". Zephyrus XXIII-XXIV: 5-17. Universidad de Salmanca.

FORTEA, J. y JORDÁ, F. (1976): "La Cueva de Les Mallaetes y los problemas del Paleolítico Superior del Mediterráneo español”. Zephyrus XXVI-XXVII: 129-166. Universidad de Salamanca.

G.E.S. DE LA S.E.M. (1985): La Cueva de Nerja. Sociedad Excursionista de Málaga. Málaga.

GILES, F., GUTIÉRREZ, J.M., MATA, E., SANTIAGO, A. y GRACIA, F.J. (1993): "Secuencia fluvial y paleolítica del río Guadalete (Cádiz). Resultados de las investigaciones hasta 1993". Investigaciones Arqueológicas en Andalucía (1985-1992). Proyectos: 221-227. Consejería de Cultura. Junta de Andalucía.Huelva.

GILES, F., SANTIAGO, A., GUTIÉRREZ, J.M., MATA, E. y AGUILERA, L. (1994): "Nuevas aportaciones a la secuencia del Paleolítico Superior en Gibraltar y su enmarque en el contexto suroccidental de la Península Ibérica". Gibraltar during the Quaternary. AEQUA Monografias 2: 91-101. Sevilla.

GIMÉNEZ, S. (1962): La Cueva de Nerja. Patronato de la Cueva de Nerja. Málaga.

- (1964): "La Cueva de Doña Trinidad, en Ardales". Miscelánea en Homenaje al Abate Henri Breuil I: $435-$ 448. Diputación Provincial de Barcelona.

GIMÉNEZ, S. y LAZA, M. (1962): "Informe de las excavaciones en la Cueva del Higuerón o del Suizo". Noticiario Arqueológico Hispánico 6: 60-67. Madrid.

GONZÁLEZ-TABLAS, F.J. (1986): "La ocupación postmagdaleniense de la Cueva de Nerja (La Sala de la Mina)". En JORDÁ, F. y PELLICER, M.,: La Prehistoria de la Cueva de Nerja (Málaga). Trabajos sobre la Cueva de Nerja no 1: 270-282. Patronato de la Cueva de Nerja. Málaga.

GRAZIOSI, P. (1974): L'Art Paléolithique de la "province méditerranéenne" et ses influences dans les temps post-paléolithiques. Werner Green Foundation for Anthropologie Research. New York.

JORDÁ, F. (1955): El Solutrense en España y sus problemas. Diputación Provincial de Asturias. Oviedo.

— (1978): "Arte de la Edad de Piedra. Primera parte". En JORDÁ y BLAZQUEZ, : Historia del Arte Hispánico I. La Antigüedad. 1. Editorial Alhambra. Madrid.

- (1985): Los 25.000 años de la Cueva de Nerja. Salamanca.

- (1986): "Primera Parte. Paleolítico Superior y Epipaleolítico". En JORDÁ, F. y PELLICER, M.: La Prehistoria de la Cueva de Nerja (Málaga) Trabajos sobre la Cueva de Nerja № 1. Patronato de la Cueva de Nerja. Málaga. 
LAMING-EMPERAIRE, A. (1968): La arqueología prehistórica. Ediciones Martínez Roca. Barcelona.

LAPLACE, G. (1966): Recherche sur l'origine et l'évolution des complexes leptolithiques. Ecole Freançaise de Rome. Mélanges d'Archéologie et d'Histoire. Suppléments 4. Roma.

- (1973): "La typologie analytique et structurale: Base rationnelle d'étude des industries lithiques et osseuses". Colloques Nationaux C.N.R.S. No 932. Banques de Donnés Archéologiques: 91-142. Marseille.

LOPEZ, P. y CACHO, C. (1979): "La Cueva del Higuerón (Málaga): Estudio de sus materiales". Trabajos de Prehistoria 36: 11-82. Madrid.

MARQUÉS, I. y RUIZ, A. (1975): "El Solutrense de la Cueva del Tajo del Jorox. Alozaina (Málaga)". Cuadernos de Prehistoria de la Universidad de Granada 1: 47-57. Universidad de Granada.

MARQUÉS, I. y FERRER, J.E. (1991): "Hallazgo de industria solutreogravetiense en el Bajondillo (Torremolinos, Málaga)". Baetica 13: 138-145. Universidad de Málaga.

MARTÍN, E. (1988): "El poblamiento neolítico en la zona oriental de la provincia de Málaga". Mainake X: 51-60. Diputación Provincial.Málaga.

- (1989): "La industria lítica tallada y el poblamiento neolítico en la zona oriental de Málaga". XIX Congreso Nacional de Arqueología: 187-196. Zaragoza.

- (1995): La secuencia del Cerro de Capellanía (Periana, Málaga) en su contexto de la Prehistoria Reciente de la Depresión de Colmenar-Periana y su contribución al estudio de las industrias líticas talladas. Tesis Doctoral. Universidad de Sevilla.

NEHREN, R. (1992): Zur Prähistorie der Maghrebländer (Marokko-Algerien-Tunesien). Verlag Philipp Von Zabern. Kommission für Allgemeine und Vergleichende Archäologie. Bonn. Mainz am Rhein.

OTTE, M. (1985): Les industries a pointes foliacees et a pointes pedonculees dans le nord-ouest europeen. Editions du Centre d'Etudes et de Documentation Archeologiques. Viroinvai.

- (1990): "Les processus de transition du Paléolithique Moyen au Supérieur". Paléolithique Moyen récent et Paléolithique Supérieur ancien en Europe. Colloque International de Nemours: 145-149. Mémoires du Musée de Préhistoire d'Ile de France no 63 . Nemours.

PERICOT, L. (1942): La Cueva del Parpalló (Gandía). C.S.I.C. Madrid.

RAMOS, J. (1986): Yacimientos líticos y poblamiento humano prehistórico del Alto Vélez (ríos Sábar y Guaro, Málaga). Tesis Doctoral. Universidad de Sevilla.

- (1988): El poblamiento prehistórico del Alto Vélez hasta la Edad del Bronce. Biblioteca Popular Malagueña. Diputación Provincial. Málaga.

- (1988-1989): "Las industrias líticas del Neolítico en Andalucía, sus implicaciones espaciales y económicas". Zephyrus XLI-XLII: 113-148. Universidad de Salamanca.

RAMOS, J., ESPEJO, M., CANTALEJO, P., MARTÍN, E., MOLINA, J.A., RAMIREZ, F., DURÁN, J.J., GRUN, R. , FORD, D. y ALCAZAR, J. (1992): Cueva de Ardales (Málaga). su recuperación y estudio. Ayuntamiento de Ardales. Málaga.

RAMOS, J., BORJA, F., SÁEZ, A., CASTAÑEDA, V., CEPILLO, J. y PÉREZ, M. (1993): "La ocupación prehistórica de la campiña litoral y banda atlántica de Cádiz. Informe de la campaña de prospecciones arqueológicas de 1992 en San Fernando". Investigaciones Arqueológicas en Andalucía. Proyectos: 353366. Junta de Andalucía. Huelva.

RAMOS, J., CASTAÑEDA, V., PÉREZ, M. y LAZARICH, M. (1994): "Las ocupaciones humanas de la Prehistoria Reciente de la campiña litoral y banda atlántica de Cádiz. Ensayo de síntesis". Gibraltar during the Quaternary. AEQUA Monografías 2: 71-90. Sevilla.

RAMOS, J., CASTAÑEDA, V., PÉREZ, M. SÁEZ, A. y CEPILLO, J., en prensa a: "Informe de la campaña de prospección de 1992 en San Fernando (Cádiz). Su enmarque en el comienzo del proyecto de investiga- 
ción: La ocupación prehistórica de la campiña litoral y banda atlántica de Cádiz". Anuario Arqueológico de Andalucía. 1992. Actividades Sistemáticas. Consejería de Cultura. Junta de Andalucía. Sevilla.

RAMOS, J., CASTAÑEDA, V., PÉREZ, M., LAZARICH, M., MARTÍNEZ, C., MONTAÑÉS, M., CALDERÓN, D. y LOZANO, J.M., En prensa b: "Estado actual del conocimiento del proyecto de investigación "La ocupación prehistórica de la campiña litoral y banda atlántica de Cádiz". Balance tras la tercera campaña de prospecciones. 1994. Conil de la Frotnera". Anuario Arqueológico de Andalucía. Actividades Ssitemáticas. 1994. Consejería de Cultura. Junta de Andalucía. Sevilla.

RAMOS, J., ESPEJO, M., CANTALEJO, P. DURÁN, J.J. y MARTÍN, E., En prensa c: "Cueva de Ardales (Málaga): Geocronología evolutiva y cambios climáticos en el Pleistoceno Superior y Holoceno. La presencia humana desde el Paleolítico Superior al Calcolítico". El Cuaternario en la Alta Andalucía. AEQUA Monografías 3. Sevilla.

RIPOLL, E. (1970): “Acerca del problema de los orígenes del arte levantino”. Valcamonica Symposium, pp. 57-67. Centro Camuno.

RIPOLL, S. (1986): El Solutrense de Cueva Ambrosio. Vélez-Blanco, Almería. Campaña de 1963. Excavaciones Arqueológicas en España 148. Madrid.

- (1988): La Cueva de Ambrosio (Almería, Spain) y su posición cronoestratigráfica en el Mediterráneo Occidental. B.A.R. International Series 462 . Oxford.

RUIZ, B. y LEIVA, J. (1980): "El taller de sílex paleolítico de El Chorro (El Burgo, Málaga)". Mainake 1: 527. Málaga.

SANCHIDRIÁN, J.L. (1981): Cueva Navarro (Cala del Moral, Málaga). Corpus Artis Rupestris. I. Paleolithica Ars. Vol. 1. Salamanca.

- (1986a): "El arte prehistórico de la Cueva de Nerja". En JORDÁ, F. y PELLICER, M.: La Prehistoria de la Cueva de Nerja (Málaga). Trabajos sobre la Cueva de Nerja no 1: 284-330. Patronato de la Cueva de Nerja. Málaga.

- (1986b): "Arte rupestre. La Cueva de La Pileta, hoy". Revista de Arqueología 66: 34-44. Madrid.

- (1991-1992): "Códigos gráficos en algunos santuarios solutrenses de Andalucía”. Zephyrus XLIV-XLV: 17-33. Universidad de Salamanca.

- (1994): "Arte prehistórico en Andalucía: tendencias actuales y perspectivas". Actas del II Congreso de Historia de Andalucía.l Prehistoria:79-103. Córdoba.

SONNEVILLE-BORDES, D., de (1960): Le Paléolithique supérieur en Périgord. Ed. Delmas. Bordeaux.

SUCH, M. (1920): Avance al estudio de la caverna "Hoyo de la Mina" en Málaga. Boletín de la Sociedad Malagueña de Ciencias. Málaga.

TESTARD, A. (1966): Essai sur le fondement de la division sexuelle du travail chez les chasseurs-cuilleurs. Editions EHESS-C.N.R.S. Cahiers de l'Homme. Paris.

- (1985): Le Comunisme Primitif. Vol 1. Economie et Ideologie. Editions de la Maison des Sciences de l'Homme. Paris.

TEXIER, J.P. y RAYNAL, J.P. (1989): “Les “sables beiges" du Nord-Ouest du Maroc: nouvelles interprétations dynamiques, chronologiques et paléoclimatiques". C.R.Acad. Sci 309: 1577-1582. Paris.

VALLESPÍ, E. (1986): "El Paleolítico Inferior y Medio en Andalucía". Actas del Congreso Homenaje a Luis Siret (1934-1984): 59-66. Cuevas del Almanzora. Junio 1984. Consejería de Cultura. Junta de Andalucía. Sevilla.

— En prensa: "I. Paleolítico y Epipaleolítico". II Congreso Internacional El Estrecho de Gibraltar. Ceuta, Noviembre 1990. U.N.E.D. Madrid.

WENIGER, G. (1982): Wildbeuter und ihre Umwelt. Ein Beitrag zum Magdalénien Südwestdeutschlands aus ökologischer und ethno-archäologischer Sicht. Verlag Archaeologica Venatoria. Institut für Urgeschichte der Universität Tübingen. Tübingen. 
WENIGER, G. (1989): “The Magdalenian in Western Central Europe: Settlement Pattern and Regionality”. Journal of World Prehistory Vol 3 № 3, pp. 323-372. Plenum Press. New York.

- (1990): "Germany at 18.000 B.P.". In SOFER, O. y GAMBLE, C., ed.: The World at 18.000 B.F., pp. 171192. London.

— (1991): “Überlegungen zur Mobilität jägerischen Gruppen im Jungpaläolithikum”. Saeculum. Jahrbuch für Universalgeschichte. München. 